ALEA, Lat. Am. J. Probab. Math. Stat. 13, 399-415 (2016)

DOI: 10.30757/ALEA.v13-16

\title{
Integrability conditions on coboundary and transfer function for limit theorems
}

\section{Davide Giraudo}

Normandie Université, Université de Rouen, Laboratoire de Mathématiques Raphaël Salem, CNRS, UMR 6085, Avenue de l'université, BP 12, Saint-Etienne du Rouvray Cedex, Normandy, France.

E-mail address: davide.giraudo1@univ-rouen.fr

URL: http://lmrs.univ-rouen.fr/Persopage/Giraudo/index_en.html

\begin{abstract}
For a measure preserving automorphism $T$ of a probability space, we provide conditions on the tail function of $g: \Omega \rightarrow \mathbb{R}$ and $g-g \circ T$ which guarantee limit theorems among the weak invariance principle, Marcinkievicz-Zygmund strong law of large numbers and the law of the iterated logarithm to hold for $f:=m+$ $g-g \circ T$, where $\left(m \circ T^{i}\right)_{i \geqslant 0}$ is a martingale differences sequence.
\end{abstract}

\section{Introduction and notations}

Let $(\Omega, \mathcal{F}, \mu)$ be a probability space and $T: \Omega \rightarrow \Omega$ be a bijective bi-measurable and measure preserving map. We assume that the dynamical system is ergodic (that is, if $T^{-1} A=A$ for some $A \in \mathcal{F}$, then $\mu(A) \in\{0,1\}$ ). If $n \geqslant 1$ is an integer and $f: \Omega \rightarrow \mathbb{R}$, we denote $S_{n}(f):=\sum_{j=0}^{n-1} f \circ T^{j}$ and for a fixed $t$, define

$$
S_{n}^{\mathrm{pl}}(f, t):=S_{[n t]}(f)+(n t-[n t]) f \circ T^{[n t]}, t \in[0,1]
$$

where $[x]$ denote the integer part of the real number $x$. Then for each $\omega \in \Omega$ and each integer $n \geqslant 1$, the map $t \mapsto S_{n}^{\mathrm{pl}}(f, t)$ is an element of the space of continuous functions on $[0,1]$, denoted by $C[0,1]$.

Let us state the limit theorems we are interested in.

Definition 1.1. Let $f: \Omega \rightarrow \mathbb{R}$ be a measurable function.

- We say that the function $f$ satisfies the invariance principle if the sequence $\left(n^{-1 / 2} S_{n}^{\mathrm{pl}}(f, \cdot)\right)_{n \geqslant 1}$ weakly converges in the space $C[0,1]$ endowed with the topology of uniform convergence to a scalar multiple of a standard Brownian motion.

Received by the editors May 5, 2016; accepted April 27, 2016.

2010 Mathematics Subject Classification. 60F17, 60G10.

Key words and phrases. invariance principle, strictly stationary processes, martingales, coboundary. 
- We say that the function $f$ satisfies the law of the iterated logarithm if there exists a constant $C(f)$ such that for almost every $\omega \in \Omega$,

$$
\limsup _{n \rightarrow+\infty} \frac{S_{n}(f)(\omega)}{\sqrt{n \log \log n}}=C(f) \text { and } \liminf _{n \rightarrow+\infty} \frac{S_{n}(f)(\omega)}{\sqrt{n \log \log n}}=-C(f) .
$$

- We say that the function $f$ satisfies the functional law of the iterated logarithm if the sequence $\left((\sqrt{n \log \log n})^{-1} S_{n}^{\mathrm{pl}}(f, \cdot)\right)_{n \geqslant 1}$ is relatively compact and the set of its limit points coincides with the set of all absolutely continuous functions $x \in C[0,1]$ such that $x(0)=0$ and $\int_{0}^{1}\left(x^{\prime}(t)\right)^{2} \mathrm{~d} t \leqslant 1$, where $x^{\prime}$ denotes the derivative of $x$ defined almost everywhere with respect to the Lebesgue measure.

- Let $1<p<2$. We say that the function $f$ satisfies the $p$-strong law of large numbers if for any $\alpha \in[1 / p, 1]$, the following holds:

$$
\forall \varepsilon>0, \quad \sum_{n=1}^{+\infty} n^{\alpha p-2} \mu\left\{\max _{1 \leqslant k \leqslant n}\left|S_{k}(f)\right| \geqslant \varepsilon n^{\alpha}\right\}<+\infty .
$$

If it is possible to find a decomposition of the function $f$

$$
f=m+g-g \circ T
$$

where $g: \Omega \rightarrow \mathbb{R}$ is a measurable function and $m$ satisfies one of the previous limit theorems, then one can wonder if we can deduce the result for $f$.

A known situation is when the sequence $\left(m \circ T^{i}\right)_{i \geqslant 0}$ is a square-integrable martingale differences sequence. A necessary and sufficient condition to have (1.4) with such an $m$ and a square integrable $g$ is known (see Theorem 2 in Volný, 1993). If $\left(m \circ T^{i}\right)_{i \geqslant 0}$ is a square-integrable martingale difference sequence, then the functional law of the iterated logarithm and the invariance principle take place.

If $1<p<2$ and $m \in \mathbb{L}^{p}$, then Theorem 5 by Dedecker and Merlevède (2007) implies that $m$ satisfies the $p$-strong law of large numbers. Actually, their results holds in a more general setting than strictly stationary sequences, as they only require a stochastic domination on the considered martingale differences sequence. A similar result as (1.3) takes place for $\alpha=1$ if we require a conditional stochastic domination (see Benoist and Quint, 2016, Theorem 2.2). A necessary and sufficient condition for (1.4) to hold with $m, g \in \mathbb{L}^{p}, 1<p<2$, is given by Volný (2006, Theorem 1), and in this case, (1.3) is satisfied (see Theorem 6 of Dedecker and Merlevède, 2007).

We call a coboundary a function of the form $g-g \circ T$, where $g: \Omega \rightarrow \mathbb{R}$ is a measurable function. The function $g$ is called a transfer function. The following result is Theorem 1 of Volný and Samek (2000). It gives a necessary and sufficient condition on the transfer function to preserve the limit theorems mentioned in the previous definition. Sufficiency for the invariance principle and the law of the iterated logarithm was established in Hall and Heyde (1980, pages 140-142).

Theorem 1.2 (The equivalence theorem,Volný and Samek, 2000). Let us suppose that for the process $\left(m \circ T^{i}\right)_{i \in \mathbb{Z}}$ the invariance principle, the law of the iterated logarithm (functional law of the iterated logarithm) respectively, holds true. Let $g$ be a measurable function and

$$
f=m+g-g \circ T .
$$

Then for the process $\left(f \circ T^{i}\right)_{i \in \mathbb{Z}}$ 
- the invariance principle holds if and only if

$$
\frac{1}{\sqrt{n}} \max _{1 \leqslant k \leqslant n}\left|g \circ T^{k}\right| \underset{n \rightarrow \infty}{\rightarrow} 0 \text { in probability; }
$$

- the law of the iterated logarithm as well as the functional law of the iterated logarithm holds if and only if

$$
\frac{1}{\sqrt{n \log \log n}} g \circ T^{n} \underset{n \rightarrow \infty}{\rightarrow} 0 \text { a.s. }
$$

Both conditions (1.6) and (1.7) take place when the function $g$ is square-integrable. If $1<p<2$, Theorem 6 in Dedecker and Merlevède (2007) shows that (1.3) holds if $g$ belongs to $\mathbb{L}^{p}$.

However, it may happen that we obtain a decomposition (1.4) where $m \in \mathbb{L}^{2}$ but the function $g$ is only integrable (see Volný and Samek (2000) for explicit counter-examples, and Esseen and Janson (1985) for a condition which guarantees the square integrability of $m$ ) and in this case, the weak invariance principle does not need to hold. We investigate conditions on the functions $t \mapsto \mu\{|g|>t\}$ and $t \mapsto \mu\{|g-g \circ T|>t\}$ which guarantee (1.6) or (1.7). In order to state these conditions in a more concise way, we introduce the so-called weak $\mathbb{L}^{q}$-spaces.

Definition 1.3. Let $q$ be a real number strictly greater than 1 . We denote by $\mathbb{L}^{q, \infty}$ the space of functions $h: \Omega \rightarrow R$ such that

$$
\|h\|_{q, \infty}^{q}:=\sup _{t>0} t^{q} \mu\{|h|>t\} \text { is finite. }
$$

The subspace of $\mathbb{L}^{q, \infty}$ which consists of functions $h$ such that $\lim _{t \rightarrow+\infty} t^{q} \mu\{|h|>t\}=0$ is denoted by $\mathbb{L}_{0}^{q, \infty}$.

\section{Main results}

In this section, we state the main results of this note. In the first subsection, we give a sufficient condition on the functions $t \mapsto \mu\{|g|>t\}$ and $t \mapsto$ $\mu\{|g-g \circ T|>t\}$ in order to preserve the weak invariance principle, the law of the iterated logarithm and the $p$-strong law of large numbers respectively. We provide projective conditions which guarantee these sufficient conditions an a martingale coboundary decomposition.

In the second subsection, we construct counter-examples which show that the found conditions are sharp when the considered dynamical system is aperiodic.

Finally, in the third subsection, we provide applications of the results of Subsection 2.1 to Bernoulli shifts.

Volný and Samek (2000) showed that the conclusion of Theorems 2.1 and 2.4 (see the next subsections) holds when $p \geqslant(r+2) / r$ and that of Theorem 2.10 when $p<(r-1) /(r-3 / 2)$. In the case $r>2$, we cannot conclude from their results if $(r-1) /(r-3 / 2) \leqslant p<(r+2) / r$, while it is the case with our conditions.

\subsection{Sufficient conditions.}

Theorem 2.1. Let $1<p<2$ and let $g: \Omega \rightarrow \mathbb{R}$ be a function such that $g \in \mathbb{L}_{0}^{p, \infty}$ and $g-g \circ T \in \mathbb{L}_{0}^{p /(p-1), \infty}$. Then for any square integrable martingale differences sequence $\left(m \circ T^{i}\right)_{i \geqslant 0}$, the function $f:=m+g-g \circ T$ satisfies the weak invariance principle in $C[0,1]$. 
A similar result has been obtained for the quenched functional central limit theorem (see Barrera et al. (2016), Corollary 7).

Corollary 2.2. Let $1<p<2$ and let $\mathcal{M}$ be a sub- $\sigma$-algebra of $\mathcal{F}$ such that $T \mathcal{M} \subset \mathcal{M}$. Assume that $f$ is an $\mathcal{M}$-measurable element of $\mathbb{L}_{0}^{p /(p-1), \infty}$ such that the following two conditions hold:

$$
\text { the sequence }\left(\mathbb{E}\left[S_{n}(f) \mid \mathcal{M}\right]\right)_{n \geqslant 1} \text { converges in } \mathbb{L}^{p, \infty} \text {; }
$$

the sequence $\left((I-U) \mathbb{E}\left[S_{n}(f) \mid \mathcal{M}\right]\right)_{n \geqslant 1}$ converges in $\mathbb{L}^{p /(p-1), \infty}$.

Then the function $f$ satisfies the invariance principle.

The conditions of Corollary 2.2 imply that $f$ admit the martingale-coboundary representation (1.4) with $m$ and $g$ integrable and $g$ satisfies (1.6). In Durieu and Volný (2008); Durieu (2009), the later condition was compared with Dedecker and Rio projective criterion (cf. Dedecker and Rio, 2000):

$$
\text { the sequence } f \mathbb{E}\left[S_{n}(f) \mid \mathcal{M}\right] \text { converges in } \mathbb{L}^{1} \text {, }
$$

which also implies that $f$ satisfies the invariance principle. It was shown that there is an example of function $f$ which satisfies the martingale-coboundary decomposition in $\mathbb{L}^{1}$ and the invariance principle but not (2.3).

Here do not assume that $f$ belongs to $\mathbb{L}^{p /(p-1)}$ and that the convergence in (2.2) holds in $\mathbb{L}^{p}$, otherwise, the function $f$ would satisfy Dedecker-Rio projective criterion. In this way, our condition is independent of (2.3).

Proposition 2.3. Let $(\Omega, \mathcal{F}, \mu)$ be a dynamical system of positive entropy. Let $1<p<2$. There exists a sub- $\sigma$-algebra $\mathcal{M}$ such that $T \mathcal{M} \subset \mathcal{M}$ and a function $f \in \mathbb{L}_{0}^{p /(p-1), \infty}$ satisfying (2.1) and (2.2) but not (2.3).

Theorem 2.4. Let $1<p<2<r$ and let $g: \Omega \rightarrow \mathbb{R}$ be a function.

(i) If $p>r /(r-1), g \in \mathbb{L}^{p, \infty}$ and $g-g \circ T \in \mathbb{L}^{r, \infty}$, then for any martingale differences sequence $\left(m \circ T^{i}\right)_{i \geqslant 0}$, the function $f:=m+g-g \circ T$ satisfies the law of the iterated logarithm;

(ii) if $p=r /(r-1), g \in \mathbb{L}^{p}$ and $g-g \circ T \in \mathbb{L}^{r}$, then for any square integrable martingale differences sequence $\left(m \circ T^{i}\right)_{i \geqslant 0}$, the function $f:=m+g-g \circ T$ satisfies the law of the iterated logarithm.

Corollary 2.5. Let $1<p<2$ and let $\mathcal{M}$ be a sub- $\sigma$-algebra of $\mathcal{F}$ such that $T \mathcal{M} \subset \mathcal{M}$. Assume that $f$ is an $\mathcal{M}$-measurable element of $\mathbb{L}^{p /(p-1)}$ such that the following two conditions hold:

$$
\text { the sequence }\left(\mathbb{E}\left[S_{n}(f) \mid \mathcal{M}\right]\right)_{n \geqslant 1} \text { converges in } \mathbb{L}^{p} \text {; }
$$

$$
\text { the sequence }\left((I-U) \mathbb{E}\left[S_{n}(f) \mid \mathcal{M}\right]\right)_{n \geqslant 1} \text { converges in } \mathbb{L}^{p /(p-1)} \text {. }
$$

Then the function $f$ satisfies the functional law of the iterated logarithms.

Remark 2.6. In Volný and Samek (2000), $\mathbb{L}^{q}$ spaces are involved. It turns out that in the setting of Theorems 2.1 and 2.4, (i), we may work with weak $\mathbb{L}^{q}$-spaces. For the case (ii) in Theorem 2.4, it is an open question to determine whether strong moments are actually needed. 
Theorem 2.7. Let $1 \leqslant q<p<r<2$ be real numbers and let $g: \Omega \rightarrow \mathbb{R}$ be a function such that $g \in \mathbb{L}^{q}$ and $g-g \circ T \in \mathbb{L}^{r}$. If $q \geqslant(p-1) r /(r-1)$, then for any martingale differences sequence $\left(m \circ T^{i}\right)_{i \geqslant 0}$ with $m \in \mathbb{L}^{p}$, the function $f:=m+g-g \circ T$ satisfies (1.3).

Corollary 2.8. Let $1<p<r<2$ and let $\mathcal{M}$ be a sub- $\sigma$-algebra of $\mathcal{F}$ such that $T \mathcal{M} \subset \mathcal{M}$. Assume that $f$ is an $\mathcal{M}$-measurable element of $\mathbb{L}^{r}$ such that the following two conditions hold:

$$
\begin{gathered}
\text { the sequence }\left(\mathbb{E}\left[S_{n}(f) \mid \mathcal{M}\right]\right)_{n \geqslant 1} \text { converges in } \mathbb{L}^{\max \{1,(p-1) r /(r-1)\}} ; \\
\text { the sequence }\left((I-U) \mathbb{E}\left[S_{n}(f) \mid \mathcal{M}\right]\right)_{n \geqslant 1} \text { converges in } \mathbb{L}^{r} .
\end{gathered}
$$

Then for each positive $\varepsilon$ and any $\alpha \in[1 / p, 1]$, we have

$$
\sum_{n=1}^{+\infty} n^{\alpha p-2} \mu\left\{\max _{1 \leqslant k \leqslant n}\left|S_{k}(f)\right| \geqslant \varepsilon n^{\alpha}\right\}<+\infty .
$$

Remark 2.9. Ergodicity of the dynamical system is required for the "only if" direction in the equivalence involving the law of the iterated logarithm of Theorem 1.2. Therefore, the results of this subsection remain valid in the non-ergodic setting.

2.2. Counter-examples. In Theorems 2.1 and 2.4, we gave a sufficient condition on $(p, r)$ for which $g \in \mathbb{L}^{p}$ and $g-g \circ T^{r} \in \mathbb{L}^{r}$ guarantees the invariance principle, and the law of iterated logarithms for $g-g \circ T$. The next results show that when this condition is not satisfied, these limit theorems may fail, which shows its sharpness.

Theorem 2.10. Assume that the dynamical system $(\Omega, \mathcal{F}, \mu, T)$ is aperiodic. Let $1 \leqslant p<2 \leqslant r$ be real numbers such that $p<r /(r-1)$. Then there exists a function $g \in \mathbb{L}^{p}$ such that $g-g \circ T \in \mathbb{L}^{r}$ and the function $g-g \circ T$ satisfies neither the invariance principle nor the law of the iterated logarithm.

We also have a similar counter-example for the $p$-strong law of large numbers.

Theorem 2.11. Assume that the dynamical system $(\Omega, \mathcal{F}, \mu, T)$ is aperiodic. Let $1<p<2$ and let $1 \leqslant q<p<r$ be real numbers such that $q<(p-1) r /(r-1)$. Then there exists a function $g \in \mathbb{L}^{q}$ such that $g-g \circ T \in \mathbb{L}^{r}$ but the sequence $\left(n^{-1 / p} S_{n}(g-g \circ T)\right)_{n \geqslant 1}$ does not converge almost surely to 0. In particular, $g-g \circ T$ does not satisfy (1.3)

2.3. Applications. In Subsection 2.1, we provided sufficient conditions for the functional central limit theorem, the law of the iterated logarithm and the $p$-law of large numbers. It is natural to try to apply these conditions to some strictly stationary sequences.

In the case of a linear process $f \circ T^{k}=\sum_{i \geqslant 0} a_{i} \varepsilon_{k-i}$, where $\left(\varepsilon_{i}\right)_{i \in \mathbb{Z}}$ is an i.i.d. centered sequence of random variables in $\mathbb{L}_{0}^{p /(p-1), \infty}, 1<p<2$, condition (2.1) implies that $f$ has a martingale coboundary decomposition in $\mathbb{L}^{q}, 1<q<p$. Since $q / 2<1$, the Marcinkievicz-Zygmund and Jensen's inequalities give the convergence of the series $\sum_{i \geqslant 0}\left(\sum_{k \geqslant i} a_{k}\right)^{1 / 2}$, which in turn implies the martingale-coboundary decomposition in $\mathbb{L}^{2}$, from which the invariance principle could have been already deduced. 
This is why we shall focus on some functionals of a particular linear process: the so-called Bernoulli shifts. Let $\left(\varepsilon_{k}\right)_{k \in \mathbb{Z}}$ by an i.i.d. sequence of random variables which take the values 0 and 1 with probability $1 / 2$. We define for $x=$ $\sum_{k=1}^{+\infty} 2^{-k-1} \varepsilon_{-k}$

$$
T^{n} x:=\sum_{k=1}^{+\infty} 2^{-k-1} \varepsilon_{n-k}
$$

The map $T$ preserves the Lebesgue measure $\lambda$ on the unit interval endowed with the Borel $\sigma$-algebra. Given a function $f:[0,1] \rightarrow \mathbb{R}$, we would like to give some sufficient conditions on the regularity of $f$ which guarantee the invariance principle, the law of the iterated logarithm and the $p$-law of large numbers.

Proposition 2.12. Let $f: \Omega \rightarrow \mathbb{R}$ be a centered function such that for some $p \in$ $(1,2), t^{p /(p-1)} \lambda\{|f|>t\}=0$ and for some $\delta>0$,

$$
\begin{gathered}
\int_{0}^{1} \int_{0}^{1} \frac{|f(x)-f(y)|^{p}}{|x-y|}\left(\log \frac{1}{|x-y|}\right)^{p-1+\delta} \mathrm{d} x \mathrm{~d} y<\infty \text { and } \\
\int_{0}^{1} \int_{0}^{1} \frac{|\widetilde{f}(x)-\widetilde{f}(y)|^{\frac{p}{p-1}}}{|x-y|}\left(\log \frac{1}{|x-y|}\right)^{\frac{1}{p-1}+\delta} \mathrm{d} x \mathrm{~d} y<\infty,
\end{gathered}
$$

where $\tilde{f}(x)=f(x)-f(x / 2) / 2-f((x+1) / 2) / 2$.

Then $f$ satisfies the invariance principle and the functional law of the iterated logarithm.

Proposition 2.13. Let $p \in(1,2)$. Let $f: \Omega \rightarrow \mathbb{R}$ be a centered function such that for some $r \in(p, 1), f$ belongs to $\mathbb{L}^{r}$ and for some $\delta>0$,

$$
\begin{gathered}
\int_{0}^{1} \int_{0}^{1} \frac{|f(x)-f(y)|^{q}}{|x-y|}\left(\log \frac{1}{|x-y|}\right)^{q-1+\delta} \mathrm{d} x \mathrm{~d} y<\infty \text { and } \\
\int_{0}^{1} \int_{0}^{1} \frac{|\widetilde{f}(x)-\widetilde{f}(y)|^{r}}{|x-y|}\left(\log \frac{1}{|x-y|}\right)^{r-1+\delta} \mathrm{d} x \mathrm{~d} y<\infty,
\end{gathered}
$$

where $q:=\max \{1,(p-1) r /(r-1)\}$ and $\widetilde{f}(x)=f(x)-f(x / 2) / 2-f((x+1) / 2) / 2$. Then $f$ satisfies the p-strong law of large numbers.

\section{Proofs}

If $h: \Omega \rightarrow \mathbb{R}$ is a measurable function, we define $M^{*}(h):=\sup _{N \geqslant 1} N^{-1}\left|S_{N}(h)\right|$.

The following lemma about Birkhoff averages will be used in the proof.

Lemma 3.1. Let $q>1$ and let $h: \Omega \rightarrow R$ be a measurable function.

(i) If $h$ belongs to $\mathbb{L}_{0}^{q, \infty}$ then the function $M^{*}(h)$ belongs to $\mathbb{L}_{0}^{q, \infty}$;

(ii) if $h$ belongs to $\mathbb{L}^{q}$, then so does $M^{*}(h)$.

Proof: By the maximal ergodic theorem, we have for each positive $t$,

$$
t \cdot \mu\left\{M^{*}(h) \geqslant t\right\} \leqslant \mathbb{E}\left[|h| \cdot \mathbf{1}\left\{M^{*}(h) \geqslant t\right\}\right] .
$$

(i) The expectation can be bounded by

$$
\int_{0}^{+\infty} \min \left\{\mu\left(A_{t}\right), s^{-q}\left\|h \cdot \mathbf{1}\left(A_{t}\right)\right\|_{q, \infty}\right\} \mathrm{d} s
$$


where

$$
A_{t}:=\left\{M^{*}(h) \geqslant t\right\} .
$$

Therefore, we infer the bound

$$
\begin{aligned}
\mathbb{E}\left[|h| \cdot \mathbf{1}\left(A_{t}\right)\right] & \leqslant \mu\left(A_{t}\right)^{1-1 / q}\left\|h \mathbf{1}\left(A_{t}\right)\right\|_{q, \infty}+ \\
& +\left\|h \cdot \mathbf{1}\left(A_{t}\right)\right\|_{q, \infty}^{q} \int_{\left\|h \cdot \mathbf{1}\left(A_{t}\right)\right\|_{q, \infty} \mu\left(A_{t}\right)^{-1 / q}}^{+\infty} s^{-q} \mathrm{~d} s \\
& =\mu\left(A_{t}\right)^{1-1 / q}\left\|h \cdot \mathbf{1}\left(A_{t}\right)\right\|_{q, \infty}+ \\
& +\left\|h \cdot \mathbf{1}\left(A_{t}\right)\right\|_{q, \infty}^{q} \frac{\left(\left\|h \cdot \mathbf{1}\left(A_{t}\right)\right\|_{q, \infty} \mu\left(A_{t}\right)^{-1 / q}\right)^{1-q}}{q-1} \\
& =\mu\left(A_{t}\right)^{1-1 / q}\left\|h \cdot \mathbf{1}\left(A_{t}\right)\right\|_{q, \infty}+\frac{\left\|h \cdot \mathbf{1}\left(A_{t}\right)\right\|_{q, \infty} \mu\left(A_{t}\right)^{1-1 / q}}{q-1} \\
& =\frac{q}{q-1} \mu\left(A_{t}\right)^{1-1 / q}\left\|h \cdot \mathbf{1}\left(A_{t}\right)\right\|_{q, \infty} \cdot
\end{aligned}
$$

Plugging this into (3.1), we obtain

$$
t \cdot \mu\left\{M^{*}(h) \geqslant t\right\}^{1 / q} \leqslant \frac{q}{q-1}\left\|h \cdot \mathbf{1}\left(A_{t}\right)\right\|_{q, \infty}
$$

hence is is enough to prove that

$$
\lim _{t \rightarrow+\infty} \sup _{s \geqslant 0} s^{q} \mu\left(\{|h| \geqslant s\} \cap A_{t}\right)=0 .
$$

To this aim, fix a positive $\varepsilon$; by assumption, there exists a positive real number $s_{0}$ such that for $s \geqslant s_{0}$, we have $s^{q} \mu\{|h| \geqslant s\} \leqslant \varepsilon$, hence

$$
\sup _{s \geqslant 0} s^{q} \mu\left(\{|h| \geqslant s\} \cap A_{t}\right) \leqslant \max \left\{\varepsilon, s_{0}^{p} \mu\left(A_{t}\right)\right\},
$$

which is smaller than $\varepsilon$ for $t$ large enough.

(ii) This follows by multiplying (3.1) by $t^{q-2}$, integrating over $[0,+\infty)$ with respect to the Lebesgue measure and switching the integrals.

This concludes the proof of Lemma 3.1.

We now give the proofs of the main results, which combine Lemma 3.1 with the ideas of Volný and Samek (2000).

\subsection{Proof of sufficient conditions.}

Proof of Theorem 2.1: In view of Theorem 1.2, we have to show that the sequence $\left(n^{-1 / 2} \max _{1 \leqslant j \leqslant n}\left|g \circ T^{j}\right|\right)_{n \geqslant 1}$ converges to 0 in probability. 
Let $\varepsilon$ be a positive fixed number. Let $k, n$ be positive integers such that $k<n$. Denoting $p_{n}:=\mu\left\{\max _{1 \leqslant j \leqslant n}\left|g \circ T^{j}\right|>2 \varepsilon n^{1 / 2}\right\}$, the following estimates take place:

$$
\begin{aligned}
p_{n} & \leqslant \mu\left\{\max _{1 \leqslant i \leqslant\left[\frac{n}{k}\right]+1} \max _{i k \leqslant j<(i+1) k}\left|g \circ T^{i k}\right|+\left|g \circ T^{j}-g \circ T^{i k}\right|>2 \varepsilon n^{1 / 2}\right\} \\
& \leqslant \mu\left\{\max _{1 \leqslant i \leqslant\left[\frac{n}{k}\right]+1}\left|g \circ T^{i k}\right|>\varepsilon \sqrt{n}\right\} \\
& +\sum_{i=1}^{\left[\frac{n}{k}\right]+1} \mu\left\{\max _{i k \leqslant j<(i+1) k}\left|g \circ T^{j}-g \circ T^{i k}\right|>\varepsilon n^{1 / 2}\right\} \\
& \leqslant\left(\left[\frac{n}{k}\right]+1\right) \mu\left\{|g|>\varepsilon n^{1 / 2}\right\}+\left(\left[\frac{n}{k}\right]+1\right) \mu\left\{\max _{0 \leqslant j<k}\left|g \circ T^{j}-g\right|>\varepsilon n^{1 / 2}\right\} \\
& \leqslant\left(\left[\frac{n}{k}\right]+1\right) \mu\left\{|g|>\varepsilon n^{1 / 2}\right\}+\left(\left[\frac{n}{k}\right]+1\right) \mu\left\{\max _{0 \leqslant j<k} \frac{1}{j}\left|S_{j}(g-g \circ T)\right|>\varepsilon \frac{n^{1 / 2}}{k}\right\} .
\end{aligned}
$$

This yields

$$
\begin{aligned}
p_{n} \leqslant\left(\left[\frac{n}{k}\right]+1\right) \mu\left\{|g|>\varepsilon n^{1 / 2}\right\} & + \\
& \left(\left[\frac{n}{k}\right]+1\right) \mu\left\{M^{*}(g-g \circ T)>\varepsilon \frac{n^{1 / 2}}{k}\right\} .
\end{aligned}
$$

By the assumption on $p$ and $r$, the inequality

$$
\frac{r / 2-1}{r-1}=\frac{r-1-r / 2}{r-1}=1-\frac{r}{2(r-1)} \geqslant 1-\frac{p}{2}
$$

takes place, hence we may choose a number $\alpha$ such that

$$
1-\frac{p}{2} \leqslant \alpha \leqslant \frac{r / 2-1}{r-1} .
$$

We now use (3.11) with $k:=\left[n^{\alpha}\right]$. This yields, for some constant $c$ depending only on $p$ and $r$ :

$$
p_{n} \leqslant c \cdot n^{1-\alpha} \mu\left\{|g|>\varepsilon n^{1 / 2}\right\}+c \cdot n^{1-\alpha} \mu\left\{M^{*}(g-g \circ T)>\varepsilon n^{1 / 2-\alpha}\right\},
$$

and, by (3.13),

$$
\begin{aligned}
p_{n} & \leqslant c n^{p / 2} \mu\left\{|g|>\varepsilon n^{1 / 2}\right\}+ \\
& +c n^{1-\alpha-r(1 / 2-\alpha)} n^{(1 / 2-\alpha) r} \mu\left\{M^{*}(g-g \circ T)>\varepsilon n^{1 / 2-\alpha}\right\} \\
& =c n^{p / 2} \mu\left\{|g|>\varepsilon n^{1 / 2}\right\}+ \\
& +c n^{1-r / 2+\alpha(r-1)} n^{(1 / 2-\alpha) r} \mu\left\{M^{*}(g-g \circ T)>\varepsilon n^{1 / 2-\alpha}\right\} \\
& \leqslant c n^{p / 2} \mu\left\{|g|>\varepsilon n^{1 / 2}\right\}+c n^{(1 / 2-\alpha) r} \mu\left\{M^{*}(g-g \circ T)>\varepsilon n^{1 / 2-\alpha}\right\} .
\end{aligned}
$$

Since $g \in \mathbb{L}_{0}^{p, \infty}$ and $g-g \circ T \in \mathbb{L}_{0}^{r, \infty}$, we conclude by item (i) of Lemma 3.1 that the sequence $\left(p_{n}\right)_{n \geqslant 1}$ converges to 0 .

This concludes the proof of Theorem 2.1. 
Proof of Corollary 2.2: Condition (2.1) implies by Volný (1993) that $f$ may be written as $f=m+g-g \circ T$, where $\left(m \circ T^{i}\right)_{i \geqslant 0}$ is a martingale differences sequence with respect to the filtration $\left(T^{-i} \mathcal{M}\right)_{i \geqslant 0}$. Since $f$ is $\mathcal{M}$-measurable, the function $g$ may be written as $\sum_{i \geqslant 0} \mathbb{E}\left[U^{i} f \mid T \mathcal{M}\right]$. Thus, by condition (2.1), we derive that $g \in \mathbb{L}^{p, \infty}$ and since each term in the series defining $g$ belongs to $\mathbb{L}_{0}^{p, \infty}$, we derive that $g$ belongs to $\mathbb{L}_{0}^{p, \infty}$. Similarly, by condition (2.2), we infer that $g-g \circ T$ belongs to $\mathbb{L}_{0}^{p /(p-1), \infty}$. Since $f \in \mathbb{L}_{0}^{p /(p-1), \infty}$, we have $m \in \mathbb{L}_{0}^{p /(p-1), \infty}$, and accounting the inequality $p /(p-1)>2$, we conclude that $m$ is square integrable. The proof is complete since we showed that $g$ satisfies the conditions of Theorem 2.1.

Proof of Proposition 2.3: We use the contruction given in Durieu and Volný (2008). There exists two independent and $T$-invariant sub- $\sigma$-algebras $\mathcal{B}$ and $\mathcal{C}$. We consider a $\mathcal{B}$-measurable function $e_{0}: \Omega \rightarrow\{-1,1\}$ such that $\mu\left(\left\{e_{0}=1\right\}\right)=\mu\left(\left\{e_{0}=-1\right\}\right)=$ $1 / 2$, and define $e_{i}:=e_{0} \circ T^{i}, i \in \mathbb{Z}$, and $\mathcal{M}:=\mathcal{C} \vee \sigma\left\{e_{i}, i \leqslant 0\right\}$ (which satisfies $T \mathcal{M} \subset \mathcal{M})$. We introduce three sequences: $\left(\theta_{k}\right)_{k \geqslant 1} \subset(0,+\infty),\left(\rho_{k}\right)_{k \geqslant 1} \subset(0,1)$ a decreasing sequence such that $\sum_{k \geqslant 1} \rho_{k}<1$ and an increasing sequence of integers $\left(N_{k}\right)_{k \geqslant 1}$. Once these sequences are fixed, we choose a decreasing sequence $\left(\varepsilon_{k}\right)_{k \geqslant 1} \subset$ $(0,1)$ such that

$$
\sum_{k \geqslant 1} \theta_{k} N_{k} \varepsilon_{k}^{1 / p}
$$

We now consider for each fixed $k \geqslant 1$ a set $A_{k} \in \mathcal{C}$ such that

(1) the sets $A_{k}$ are mutually disjoint;

(2) $\left(1-\sum_{i \geqslant 1} \rho_{i}\right) \frac{\rho_{k}}{2} \leqslant \mu\left(A_{k}\right) \leqslant \rho_{k}$ for all $k \geqslant 1$;

(3) for each $k \geqslant 1$ and all $i, j \in\left\{0, \ldots, N_{k}+1\right\}, \mu\left(T^{-i} A_{k} \Delta T^{-j} A_{k}\right) \leqslant \varepsilon_{k}$.

The existence of such a sequence of sets as well as that of $\mathcal{B}$ and $\mathcal{C}$ is explained in Durieu and Volný (2008).

The function $f$ is defined by

$$
f=\sum_{k=1}^{+\infty} \theta_{k} e_{-N_{k}} \mathbf{1}\left(A_{k}\right)
$$

Assume that the sequence $\left(\theta_{k}\right)_{k \geqslant 1}$ is increasing and $\theta_{k} \rightarrow+\infty$. The function $f$ belongs to $\mathbb{L}_{0}^{p /(p-1), \infty}$ if

$$
\lim _{k \rightarrow+\infty} \theta_{k+1}^{p /(p-1)} \sum_{i \geqslant k} \rho_{i}=0
$$

Now, we have

$$
\begin{aligned}
\mathbb{E}\left[S_{n}(f) \mid \mathcal{M}\right] & =\sum_{k=1}^{+\infty} \theta_{k} \sum_{i=1}^{\min \left\{n, N_{k}\right\}} e_{-N_{k}+i} \mathbf{1}\left(A_{k}\right)+ \\
& \sum_{k=1}^{+\infty} \theta_{k} \sum_{i=1}^{\min \left\{n, N_{k}\right\}} e_{-N_{k}+i}\left(\mathbf{1}\left(T^{-i} A_{k} \backslash A_{k}\right)-\mathbf{1}\left(A_{k} \backslash T^{-i} A_{k}\right)\right),
\end{aligned}
$$

and since

$$
\left\|\sum_{i=1}^{\min \left\{n, N_{k}\right\}} e_{-N_{k}+i}\left(\mathbf{1}\left(T^{-i} A_{k} \backslash A_{k}\right)-\mathbf{1}\left(A_{k} \backslash T^{-i} A_{k}\right)\right)\right\|_{p} \leqslant N_{k} \varepsilon_{k}^{1 / p},
$$


the sequence $\left(\mathbb{E}\left[S_{n}(f) \mid \mathcal{M}\right]\right)_{n \geqslant 1}$ converges in $\mathbb{L}^{p}$ if

$$
\lim _{m, n \rightarrow \infty} \mathbb{E}\left|\sum_{k=1}^{+\infty} \theta_{k} \sum_{i=\min \left\{m, N_{k}\right\}}^{\min \left\{n, N_{k}\right\}} e_{-N_{k}+i} \mathbf{1}\left(A_{k}\right)\right|^{p}=0 .
$$

Since the family $\left(A_{k}\right)_{k \geqslant 1}$ is disjoint, (3.23) is equivalent to

$$
\lim _{m, n \rightarrow \infty} \sum_{k=1}^{+\infty} \theta_{k}^{p} \mathbb{E}\left|\sum_{i=\min \left\{m, N_{k}\right\}}^{\min \left\{n, N_{k}\right\}} e_{-N_{k}+i}\right|^{p} \rho_{k}=0,
$$

which is implied (by Marcinkievicz-Zygmund inequality) by

$$
\sum_{k=1}^{+\infty} \theta_{k}^{p} N_{k}^{p / 2} \rho_{k}
$$

Notice also that

$$
\begin{aligned}
(I-U) \mathbb{E} & {\left[S_{n}(f) \mid \mathcal{M}\right]=\sum_{k=1}^{+\infty} \theta_{k}(I-U) \sum_{i=1}^{\min \left\{n, N_{k}\right\}} e_{-N_{k}+i} \mathbf{1}\left(T^{-i} A_{k}\right) } \\
= & \sum_{k=1}^{+\infty} \theta_{k}\left(e_{-N_{k}+1} \mathbf{1}\left(T^{-1} A_{k}\right)-e_{-N_{k}+\min \left\{n, N_{k}\right\}} \mathbf{1}\left(T^{-\min \left\{n, N_{k}\right\}} A_{k}\right)\right),
\end{aligned}
$$

hence the sequence $\left((I-U) \mathbb{E}\left[S_{n}(f) \mid T \mathcal{M}\right]\right)_{n \geqslant 1}$ converges in $\mathbb{L}^{p /(p-1), \infty}$ as long as

$$
\lim _{k \rightarrow+\infty} \theta_{k+1}^{p /(p-1)} \sum_{i \geqslant k} \rho_{i}=0 .
$$

By Proposition 3 of Durieu and Volný (2008), the function $f$ defined by (3.19) satisfies (2.3) if and only if $\sum_{k=1}^{+\infty} \theta_{k}^{2} \sqrt{N_{k}} \rho_{k}<\infty$. We thus have to takes sequences $\left(\theta_{k}\right)_{k \geqslant 1},\left(\rho_{k}\right)_{k \geqslant 1}$ and $\left(N_{k}\right)_{k \geqslant 1}$ such that $\left(\theta_{k}\right)_{k \geqslant 1}$ is increasing, (3.25) and (3.26) hold but $\sum_{k=1}^{+\infty} \theta_{k}^{2} \sqrt{N_{k}} \rho_{k}=+\infty$. Such a selection is possible; for example, take

$$
\theta_{k}:=\frac{2^{k(p-1) / p}}{(\log k)^{2 / p}}, \quad N_{k}:=\left[\frac{2^{2 k(2-p) / p}}{k^{2 / p}}\right], \quad \rho_{k}:=2^{-k} .
$$

Proof of Theorem 2.4: In view of Theorem 1.2, we have to prove that the convergence in (1.7) takes place. Let $\alpha \in(0,1)$ be a number which will be specified later. We define

$$
m_{j}:=\sum_{i=1}^{j-1}\left[i^{\alpha}\right], j \geqslant 1 .
$$

Notice that for some constant $\kappa$ depending only on $\alpha$, we have

$$
\frac{j^{\alpha+1}}{\kappa} \leqslant m_{j} \leqslant \kappa j^{\alpha+1}, j \geqslant 1
$$

By the Borel-Cantelli lemma, we have to prove the convergence of the series

$$
\sum_{j=1}^{+\infty} p_{j}, \text { with } p_{j}:=\mu\left\{\max _{0 \leqslant i \leqslant\left[j^{\alpha}\right]} \frac{1}{\sqrt{m_{j} \log \log m_{j}}}\left|g \circ T^{m_{j}+i}\right|>\varepsilon\right\}
$$


for each positive $\varepsilon$. To this aim, we start from the inequalities

$$
\begin{aligned}
p_{j} & \leqslant \mu\left\{\frac{1}{\sqrt{m_{j} \log \log m_{j}}}\left|g \circ T^{m_{j}}\right|>\varepsilon / 2\right\}+ \\
& +\mu\left\{\max _{0 \leqslant i \leqslant\left[j^{\alpha}\right]} \frac{1}{\sqrt{m_{j} \log \log m_{j}}}\left|g \circ T^{m_{j}+i}-T^{m_{j}}\right|>\varepsilon / 2\right\} \\
& =\mu\left\{\frac{1}{\sqrt{m_{j} \log \log m_{j}}}|g|>\varepsilon / 2\right\}+ \\
& +\mu\left\{\max _{0 \leqslant i \leqslant\left[j^{\alpha}\right]} \frac{1}{\sqrt{m_{j} \log \log m_{j}}}\left|g \circ T^{i}-g\right|>\varepsilon / 2\right\},
\end{aligned}
$$

from which we infer

$$
\begin{aligned}
& p_{j} \leqslant \mu\left\{\frac{1}{\sqrt{m_{j} \log \log m_{j}}}|g|>\varepsilon / 2\right\}+ \\
&+\mu\left\{\frac{1}{\sqrt{m_{j} \log \log m_{j}}} M^{*}(g-g \circ T)>\frac{\varepsilon}{2\left[j^{\alpha}\right]}\right\} .
\end{aligned}
$$

(i) Assume that $p>r /(r-1)$. Using the definition of $\|\cdot\|_{p, \infty}$ and inequality (3.29), we obtain

$$
\mu\left\{\frac{1}{\sqrt{m_{j} \log \log m_{j}}}|g|>\varepsilon / 2\right\} \leqslant c(p, \varepsilon, \alpha) \kappa^{p / 2}\|g\|_{p, \infty}^{p} \frac{1}{j^{(\alpha+1) p / 2}},
$$

where $c(p, \varepsilon, \alpha)$ is independent of $j$. Using (3.29) and (3.8), we derive

$$
\begin{aligned}
& \mu\left\{\frac{1}{\sqrt{m_{j} \log \log m_{j}}} M^{*}(g-g \circ T)>\frac{\varepsilon}{2\left[j^{\alpha}\right]}\right\} \leqslant \\
& \quad \leqslant c(r, \varepsilon, \alpha) \kappa^{r / 2}\|g-g \circ T\|_{r, \infty}^{r} j^{\alpha r-(\alpha+1) r / 2}
\end{aligned}
$$

where $c(r, \varepsilon, \alpha)$ is independent of $j$.

Combining (3.31), (3.32) and (3.33), we deduce the upper bound

$$
p_{j} \leqslant c(p, r, \alpha, \varepsilon, g)\left(\frac{1}{j^{(\alpha+1) p / 2}}+\frac{1}{j^{(1-\alpha) r / 2}}\right) .
$$

We have to take $\alpha$ such that

$$
(\alpha+1) p / 2>1 \text { and }(1-\alpha) r / 2>1 .
$$

This is equivalent to

$$
\alpha>2 / p-1 \text { and } \alpha<1-2 / r .
$$

Since $2 / p-1<1-2 / r$, inequalities (3.35) are satisfied and in view of (3.34), the series defined by (3.30) is convergent for any positive $\varepsilon$. This conclude part (i) of Theorem 2.4. 
(ii) Assume that $p=r /(r-1)$. We pick $\alpha:=2 / p-1=1-2 / r$. In this case, for some constant $c$ depending only on $p$ and $r$, the inequality

$$
\begin{aligned}
p_{j} & \leqslant \mu\left\{|g|>c \varepsilon j^{1 / p}\right\}+\mu\left\{M^{*}(g-g \circ T)>\varepsilon c j^{-\alpha+(\alpha+1) / 2}\right\} \\
& =\mu\left\{|g|>c \varepsilon j^{1 / p}\right\}+\mu\left\{M^{*}(g-g \circ T)>\varepsilon c j^{(1-\alpha) / 2}\right\} \\
& =\mu\left\{|g|>c \varepsilon j^{1 / p}\right\}+\mu\left\{M^{*}(g-g \circ T)>\varepsilon c j^{1 / r}\right\}
\end{aligned}
$$

takes place. By item (ii) of Lemma 3.1, we conclude that the series defined by (3.30) is convergent for any positive $\varepsilon$ and this concludes part (ii) of Theorem 2.4, hence the proof of Theorem 2.4.

Proof of Corollary 2.5: Like in the proof of Corollary 2.2, we derive that $f$ admits a martingale-coboundary decomposition with $g$ in $\mathbb{L}^{p}$ and $m, g-g \circ T \in \mathbb{L}^{p /(p-1)}$. We thus may apply Theorem 2.4 to conclude that $f$ satisfies the functional law of the iteratd logarithms.

Proof of Theorem 2.7: Let us fix a positive $\varepsilon$ and $\alpha \in[1 / p, 1]$. Let $1 \leqslant k<n$ be integers. By similar inequalities which leaded to (3.11) (we replace the exponent $1 / 2$ by $\alpha$ ), we derive

$$
\begin{aligned}
\mu\left\{\max _{1 \leqslant j \leqslant n}\left|g-g \circ T^{j}\right|>\varepsilon n^{\alpha}\right\} & \leqslant 2\left(\left[\frac{n}{k}\right]+1\right) \mu\left\{|g|>\varepsilon n^{\alpha} / 4\right\}+ \\
+ & 2\left(\left[\frac{n}{k}\right]+1\right) \mu\left\{M^{*}(g-g \circ T)>\frac{\varepsilon n^{\alpha}}{2 k}\right\} .
\end{aligned}
$$

Let us choose $k:=\left[n^{\beta}\right]$, where

$$
(p-q) \alpha \leqslant \beta \leqslant \alpha(r-p) /(r-1)
$$

(the existence of such a $\beta$ is guaranted by the assumptions on $p, q$ and $r$ ). Then it suffices to check that for each positive constant $c$, the series

$$
\begin{gathered}
\Sigma_{1}:=\sum_{n=1}^{+\infty} n^{p \alpha-1-\beta} \mu\left\{|g| \geqslant c n^{\alpha}\right\} \text { and } \\
\Sigma_{2}:=\sum_{n=1}^{+\infty} n^{p \alpha-1-\beta} \mu\left\{M^{*}(g-g \circ T) \geqslant c n^{\alpha-\beta}\right\}
\end{gathered}
$$

are convergent. The convergence of $\Sigma_{1}$ is equivalent to the integrability of the function $|g|^{p-\beta / \alpha}$; this holds since (3.41) implies $q \geqslant p-\beta / \alpha$.

Note that the second series converges if

$$
\mathbb{E}\left[\left(M^{*}(g-g \circ T)\right)^{\frac{p \alpha-\beta}{\alpha-\beta}}\right]<+\infty .
$$

Notice that inequality (3.41) implies that $(p \alpha-\beta) /(\alpha-\beta) \leqslant r$, hence we derive the convergence of $S_{2}$ by item (ii) of Lemma 3.1 (with the exponent $(p \alpha-\beta) /(\alpha-\beta)>1$ since $p>1)$.

This concludes the proof of Theorem 2.7. 
Proof of Corollary 2.8: Like in the proof of Corollaries 2.2 and 2.5, we derive that $f$ admits a martingale-coboundary decomposition with $g \in \mathbb{L}^{(p-1) r /(r-1)}$ and $m, g-$ $g \circ T \in \mathbb{L}^{r}$. By Theorem 2.7, we derive that for each positive $\varepsilon$ and any $\alpha \in[1 / p, 1]$, (2.8) takes place.

\subsection{Counter-examples.}

Proof of Theorem 2.10: We recall the construction given in the proof of Theorem 3 of Volný and Samek (2000). We choose a real number $\alpha$ such that

$$
\frac{r-2}{2(r-1)}<\alpha<1-\frac{p}{2}
$$

This is possible because

$$
\begin{aligned}
1-\frac{p}{2}-\frac{r-2}{2(r-1)}=\frac{1}{2}(2- & \left.p-\frac{r-1-1}{r-1}\right)= \\
= & \frac{1}{2}\left(1-p+\frac{1}{r-1}\right)=\frac{1}{2}\left(\frac{r}{r-1}-p\right)>0 .
\end{aligned}
$$

For each $i \geqslant 1$, we define $n_{i}:=2^{i}$ and $k_{i}:=\left[2^{i \alpha}\right]$. By the Rokhlin lemma (see Kakutani, 1943; Rohlin, 1948), one can find a set $A_{i} \in \mathcal{F}$ such that

sets $A_{i}, T A_{i}, \ldots, T^{n_{i}-1} A_{i}$ are pairwise disjoint and

$$
\mu\left(\bigcup_{j=0}^{n_{i}-1} T^{j} A_{i}\right)>1 / 2
$$

In particular, the quantity $\mu\left(A_{i}\right)$ can be bounded as follows:

$$
\frac{1}{2 n_{i}} \leqslant \mu\left(A_{i}\right) \leqslant \frac{1}{n_{i}}
$$

We then define for $i \geqslant 1$,

$$
g_{i}:=\frac{\sqrt{n_{i} \log \log n_{i}}}{k_{i}}\left(\sum_{j=1}^{k_{i}} j \mathbf{1}\left(T^{n_{i}-j} A_{i}\right)+\sum_{j=k_{i}+1}^{2 k_{i}-1}\left(2 k_{i}-j\right) \mathbf{1}\left(T^{n_{i}-j} A_{i}\right)\right)
$$

and $g:=\sum_{i=i_{0}}^{+\infty} g_{i}$, where $i_{0}$ is such that $2 k_{i}<n_{i}$ for each $i \geqslant i_{0}$. By the BorelCantelli lemma, since $\mu\left\{g_{i} \neq 0\right\} \leqslant 2 k_{i} / n_{i} \leqslant 2^{1-(1-\alpha) i}$, the series which defines $g$ is almost surely convergent.

Since it has been shown in Volný and Samek (2000) that the function $f$ satisfies neither the invariance principle nor the law of the iterated logarithm, it remains to prove that the constructed function $g$ belongs to $\mathbb{L}^{p}$ and that the coboundary $g-g \circ T$ belongs to $\mathbb{L}^{r}$.

By (3.47) and (3.50), the equality

$$
\left|g_{i}\right|^{p}=\left(\frac{\sqrt{n_{i} \log \log n_{i}}}{k_{i}}\right)^{p}\left(\sum_{j=1}^{k_{i}} j^{p} \mathbf{1}\left(T^{n_{i}-j} A_{i}\right)+\sum_{j=k_{i}+1}^{2 k_{i}-1}\left(2 k_{i}-j\right)^{p} \mathbf{1}\left(T^{n_{i}-j} A_{i}\right)\right)
$$


takes place, hence integrating and accounting (3.49), we derive the estimates

$$
\begin{aligned}
\mathbb{E}\left|g_{i}\right|^{p} & \left.\leqslant\left(\frac{\sqrt{n_{i} \log \log n_{i}}}{k_{i}}\right)^{p}\left(\sum_{j=1}^{k_{i}} j^{p}+\sum_{j=k_{i}+1}^{2 k_{i}-1}\left(2 k_{i}-j\right)^{p}\right)\right) \frac{1}{n_{i}} \\
& \leqslant 2 \frac{n_{i}^{p / 2-1}\left(\log \log n_{i}\right)^{p / 2}}{k_{i}^{p}} k_{i}^{p+1} \\
& =2 n_{i}^{p / 2-1}\left(\log \log n_{i}\right)^{p / 2} k_{i},
\end{aligned}
$$

hence

$$
\left\|g_{i}\right\|_{p} \leqslant 2^{1 / p} n_{i}^{1 / 2-1 / p}\left(\log \log n_{i}\right)^{1 / 2} k_{i}^{1 / p}
$$

By definition of $n_{i}$ and $k_{i}$, one can find a constant $c$ depending only on $\alpha$ (hence on $p$ and $r$ ) such that for $i$ large enough,

$$
\left\|g_{i}\right\|_{p} \leqslant c \cdot 2^{i(1 / 2-1 / p)}(\log i)^{1 / 2} \cdot 2^{i \alpha / p}=c \cdot(\log i)^{1 / 2} \cdot 2^{i(\alpha-1+p / 2) / p} .
$$

By (3.45), the series $\sum_{i=1}^{+\infty}(\log i)^{1 / 2} \cdot 2^{i(\alpha-1+p / 2) / p}$ is convergent, and we conclude by (3.56) that $g$ belongs to $\mathbb{L}^{p}$.

It is proved in Volný and Samek (2000) that by construction, the equality

$$
\left|g_{i}-g_{i} \circ T\right|=\frac{\sqrt{n_{i} \log \log n_{i}}}{k_{i}} \cdot \mathbf{1}\left(\bigcup_{j=1}^{2 k_{i}} T^{n_{i}-j} A_{i}\right)
$$

holds. By (3.47) and (3.50), we have

$$
\left\|g_{i}-g_{i} \circ T\right\|_{r} \leqslant \frac{\sqrt{n_{i} \log \log n_{i}}}{k_{i}}\left(\frac{2 k_{i}}{n_{i}}\right)^{1 / r}
$$

hence by the definition of $n_{i}$ and $k_{i}$, we have for $i$ large enough and a constant $c$ depending only on $\alpha$,

$$
\left\|g_{i}-g_{i} \circ T\right\|_{r} \leqslant c \cdot 2^{i(1 / 2-1 / r)} 2^{i \alpha(1 / r-1)}(\log i)^{1 / 2},
$$

from which we infer (by (3.45)) the convergence of the series $\sum_{i=1}^{+\infty}\left\|g_{i}-g_{i} \circ T\right\|_{r}$ hence the fact that the function $g-g \circ T$ belongs to $\mathbb{L}^{r}$.

This concludes the proof of Theorem 2.10.

Proof of Theorem 2.11: The construction is similar to that of the proof of Theorem 2.10.

For each $i \geqslant 1$, we define $n_{i}:=2^{i}$ and $k_{i}:=\left[2^{i \beta}\right]$, where $\beta$ satisfies

$$
\frac{r-p}{p(r-1)}<\beta<1-\frac{q}{p} \text {. }
$$

Such a choice is possible since

$$
p-q-\frac{r-p}{r-1}>p-(p-1) \frac{r}{r-1}-\frac{r-p}{r-1}=\frac{p(r-1)-(p-1) r-r+p}{r-1}=0 .
$$

We take a set $A_{i} \in \mathcal{F}$ such that (3.47) and (3.48) hold. We then define for $i \geqslant 1$,

$$
g_{i}:=\frac{n_{i}^{1 / p}}{k_{i}}\left(\sum_{j=1}^{k_{i}} j \mathbf{1}\left(T^{n_{i}-j} A_{i}\right)+\sum_{j=k_{i}+1}^{2 k_{i}-1}\left(2 k_{i}-j\right) \mathbf{1}\left(T^{n_{i}-j} A_{i}\right)\right),
$$

and $g:=\sum_{i=i_{0}}^{+\infty} g_{i}$, where $i_{0}$ is such that $2 k_{i}<n_{i}$ for each $i \geqslant i_{0}$. 
The proof will be complete if we show the following three assertions:

(1) the function $g$ belongs to $\mathbb{L}^{q}$;

(2) the function $g-g \circ T$ belongs to $\mathbb{L}^{r}$;

(3) the sequence $\left(n^{-1 / p} g \circ T^{n}\right)_{n \geqslant 1}$ does not converge almost surely to 0 .

The first two items follow by completely similar computations as in the proof of Theorem 2.10. To show the last item, we notice that the sequence $\left(2^{-i / p} \max _{2^{i} \leqslant l \leqslant 2^{i+1}} g \circ\right.$ $\left.T^{l}\right)_{i \geqslant 1}$ does not converge to 0 in probability. To see this, one can note that

$$
\begin{aligned}
\mu\left\{2^{-i / p} \max _{2^{i} \leqslant l \leqslant 2^{i+1}} g \circ T^{l} \geqslant 1\right\} \geqslant & \\
\mu & \left\{2^{-i / p} \max _{2^{i} \leqslant l \leqslant 2^{i+1}} g_{i} \circ T^{l} \geqslant 1\right\} \geqslant \mu\left(\bigcup_{j=1}^{n_{i}-k_{i}} T^{j}\left(A_{i}\right)\right) \geqslant \frac{1}{2} .
\end{aligned}
$$

Indeed, since $g_{j}$ is non-negative, we have $g_{i} \leqslant g$, which gives the first inequality of (3.63). For the second, notice that since $n_{i}=2^{i}$,

$$
\begin{aligned}
\left\{2^{-i / p} \max _{0 \leqslant l \leqslant 2^{i}} g_{i} \circ T^{l} \geqslant 1\right\} & =\left\{\max _{0 \leqslant l \leqslant 2^{i}} \sum_{j=1}^{k_{i}} \frac{j}{k_{i}} \mathbf{1}\left(T^{n_{i}-j} A_{i}\right) \circ T^{l} \geqslant 1\right\} \\
& \supset \bigcup_{l=0}^{2^{i}}\left\{\omega \mid T^{l} \omega \in T^{n_{i}-k_{i}} A_{i}\right\} \supset \bigcup_{j=1}^{n_{i}-k_{i}} T^{j}\left(A_{i}\right) .
\end{aligned}
$$

This finishes the proof of Theorem 2.11.

3.3. Applications. For the context, we refer the reader to Subsection 2.3. We define $\mathcal{M}:=\sigma\left(\varepsilon_{i}, i \leqslant 0\right)$.

The proof of Propositions 2.12 and 2.13 will follow from Corollaries 2.2, 2.5 and 2.8 and the following intermediate step.

Lemma 3.2. Let $q>1$. Then for each centered $f:[0,1] \rightarrow \mathbb{R}$ and each $n \geqslant 1$, the following inequalities hold:

$$
\begin{gathered}
\left\|\mathbb{E}\left[f \circ T^{n} \mid \mathcal{M}\right]\right\|_{q}^{q} \leqslant 2^{n} \int_{0}^{1} \int_{0}^{1} 1\left\{|x-y| \leqslant 2^{-n}\right\}|f(x)-f(y)|^{p} \mathrm{~d} x \mathrm{~d} y \\
\left\|(I-U) \mathbb{E}\left[f \circ T^{n} \mid \mathcal{M}\right]\right\|_{q}^{q} \leqslant \\
2 \int_{0}^{1} \int_{0}^{1} 1\left\{|x-y| \leqslant 2^{-n}\right\}|\tilde{f}(x)-\tilde{f}(y)|^{q} \mathrm{~d} x \mathrm{~d} y<\infty,
\end{gathered}
$$

where $\widetilde{f}(x)=f(x)-f(x / 2) / 2-f((x+1) / 2) / 2$.

Proof: Following Maxwell and Woodroofe (2000), we have for each $x \in[0,1]$,

$$
\mathbb{E}\left[f \circ T^{n} \mid \mathcal{M}\right](x)=2^{-n} \sum_{j=0}^{2^{n}-1} \int_{0}^{1}\left[f\left(\frac{x+j}{2^{n}}\right)-f\left(\frac{y+j}{2^{n}}\right)\right] \mathrm{d} y,
$$

hence by Jensen's inequality,

$$
\left\|\mathbb{E}\left[f \circ T^{n} \mid \mathcal{M}\right]\right\|_{q}^{q} \leqslant 2^{n} \int_{0}^{1} \int_{0}^{1} \mathbf{1}\left\{|x-y| \leqslant 2^{-n}\right\}|f(x)-f(y)|^{q} \mathrm{~d} x \mathrm{~d} y .
$$


Since

$$
(I-U) \mathbb{E}\left[f \circ T^{n} \mid \mathcal{M}\right](x)=2^{-n} \sum_{j=0}^{2^{n}-1} \int_{0}^{1}\left[\tilde{f}\left(\frac{x+j}{2^{n}}\right)-\tilde{f}\left(\frac{y+j}{2^{n}}\right)\right] \mathrm{d} y,
$$

we prove (3.67) in a similar way.

Lemma 3.3. Let $q>1$. If for some positive $\delta$, we have

$$
\int_{0}^{1} \int_{0}^{1} \frac{|f(x)-f(y)|^{q}}{|x-y|}\left(\log \frac{1}{|x-y|}\right)^{q-1+\delta} \mathrm{d} x \mathrm{~d} y<\infty
$$

then the series $\sum_{n \geqslant 1}\left\|\mathbb{E}\left[f \circ T^{n} \mid \mathcal{M}\right]\right\|_{q}$ converges.

A similar sufficient condition can be stated for the convergence of the series $\sum_{n \geqslant 1}\left\|(I-U) \mathbb{E}\left[f \circ T^{n} \mid \mathcal{M}\right]\right\|_{q}$.

Proof of Lemma 3.3: In view of inequality (3.66), we have to prove that

$$
\sum_{n=1}^{+\infty}\left(2^{n} \int_{0}^{1} \int_{0}^{1} \mathbf{1}\left\{|x-y| \leqslant 2^{-n}\right\}|f(x)-f(y)|^{q} \mathrm{~d} x \mathrm{~d} y\right)^{1 / q}<+\infty .
$$

To this aim, we define $\beta:=(q-1+\delta) / q$ and bound, by Hölder's inequality,

$$
\left(\sum_{n=1}^{+\infty} n^{\beta q} 2^{n} \int_{0}^{1} \int_{0}^{1} \mathbf{1}\left\{|x-y| \leqslant 2^{-n}\right\}|f(x)-f(y)|^{q} \mathrm{~d} x \mathrm{~d} y\right)\left(\sum_{n=1}^{+\infty} n^{-\beta q /(q-1)}\right)^{1-1 / q}<+\infty
$$

and since $\beta q /(q-1)=(q-1+\delta) /(q-1)>1$, it suffices to show that the series $\sum_{n=1}^{+\infty} n^{\beta q} 2^{n} \int_{0}^{1} \int_{0}^{1} \mathbf{1}\left\{|x-y| \leqslant 2^{-n}\right\}|f(x)-f(y)|^{q} \mathrm{~d} x \mathrm{~d} y$ converges. For a fixed $t \in$ $[0,1]$, we have

$$
\sum_{n=1}^{+\infty} n^{\beta q} 2^{n} \mathbf{1}\left\{t \leqslant 2^{-n}\right\}=\sum_{n=1}^{\log _{2}(1 / t)} n^{\beta q} 2^{n} \leqslant \frac{2}{t}\left(\log _{2}(1 / t)\right)^{\beta q}
$$

from which the convergence of $\sum_{n \geqslant 1}\left\|\mathbb{E}\left[f \circ T^{n} \mid \mathcal{M}\right]\right\|_{q}$ follows.

\section{Acknowledgements}

The author would like to thank anonymous referees for their comments, which led to an improvement of the presentation of the paper.

\section{References}

D. Barrera, C. Peligrad and M. Peligrad. On the functional CLT for stationary Markov chains started at a point. Stochastic Process. Appl. 126 (7), 1885-1900 (2016). MR3483741.

Y. Benoist and J.-F. Quint. Central limit theorem for linear groups. Ann. Probab. 44 (2), 1308-1340 (2016). MR3474473.

J. Dedecker and F. Merlevède. Convergence rates in the law of large numbers for Banach-valued dependent variables. Teor. Veroyatn. Primen. 52 (3), 562-587 (2007). MR2743029.

J. Dedecker and E. Rio. On the functional central limit theorem for stationary processes. Ann. Inst. H. Poincaré Probab. Statist. 36 (1), 1-34 (2000). MR1743095. 
O. Durieu. Independence of four projective criteria for the weak invariance principle. ALEA Lat. Am. J. Probab. Math. Stat. 5, 21-26 (2009). MR2475604.

O. Durieu and D. Volný. Comparison between criteria leading to the weak invariance principle. Ann. Inst. Henri Poincaré Probab. Stat. 44 (2), 324-340 (2008). MR2446326.

C.-G. Esseen and S. Janson. On moment conditions for normed sums of independent variables and martingale differences. Stochastic Process. Appl. 19 (1), 173-182 (1985). MR780729.

P. Hall and C. C. Heyde. Martingale limit theory and its application. Academic Press, Inc. [Harcourt Brace Jovanovich, Publishers], New York-London (1980). ISBN 0-12-319350-8. Probability and Mathematical Statistics. MR624435.

S. Kakutani. Induced measure preserving transformations. Proc. Imp. Acad. Tokyo 19, 635-641 (1943). MR0014222.

M. Maxwell and M. Woodroofe. Central limit theorems for additive functionals of Markov chains. Ann. Probab. 28 (2), 713-724 (2000). MR1782272.

V. Rohlin. A "general" measure-preserving transformation is not mixing. Doklady Akad. Nauk SSSR (N.S.) 60, 349-351 (1948). MR0024503.

D. Volný. Approximating martingales and the central limit theorem for strictly stationary processes. Stochastic Process. Appl. 44 (1), 41-74 (1993). MR1198662.

D. Volný. Martingale approximation of non-stationary stochastic processes. Stoch. Dyn. 6 (2), 173-183 (2006). MR2239087.

D. Volný and P. Samek. On the invariance principle and the law of iterated logarithm for stationary processes. In Mathematical physics and stochastic analysis (Lisbon, 1998), pages 424-438. World Sci. Publ., River Edge, NJ (2000). MR1893125. 